\section{GENOME EDITING}

\section{A new CRISPR scissor}

Nature 566, 218-223 (2019)

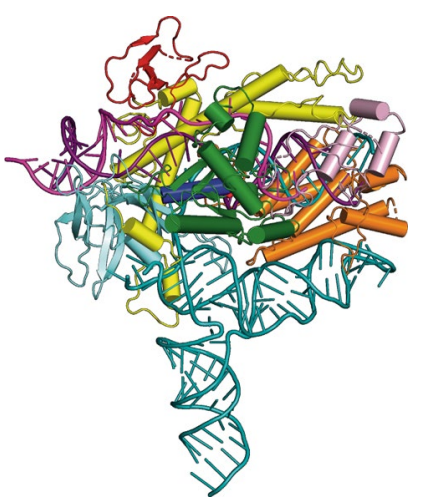

Credit: Nature

CasX was initially identified from groundwater samples via metagenomics analysis. Although it contained no similarity to other CRISPR-Cas9 enzymes, Liu et al. investigated whether CasX might act as a genome-editing enzyme. Biochemical and structural analyses of CasX from Deltaproteobacteria (DpbCasX) demonstrated that DpbCasX could cleave double-stranded DNA adjacent to a TTCN protospacer-adjacent motif with the assistance of sgRNA, and generate DNA products with staggered ends. A deactivated DpbCasX maintains RNA-guided DNAbinding ability but lacks cleavage activity for use in a CRISPR interference system. Structural analysis revealed that DpbCasX contains a unique nontargetstrand-binding (NTSB) domain and a target-strand-loading (TSL) domain. The NTSB domain is responsible for initiating
DNA duplex unwinding, and the TSL domain helps to bend an RNA-DNA hybrid duplex into the RuvC domain active site for cleavage of the target DNA strand. Despite the modest genome editing efficiency of CasX compared to SpCas9 in mammalian cells, the mechanistic insights revealed in this study pave the way for using CasX for genome editing and therapeutic applications.

https://doi.org/10.1038/s41589-019-0261-2

\section{CARDIAC REGENERATION}

\section{A broken heart can mend}

Dev. Cell https://doi.org/10.1016/j.

devcel.2019.01.001 (2019)

Adult zebrafish hearts undergo vigorous regeneration upon injury through dedifferentiation and proliferation of cardiomyocytes. Though cyclin-dependent kinases and ErbB2 have been identified as regulators of cardiomyocyte proliferation, large-scale genetic identification of related mediators has remained difficult. Han et al. used a fluorescent ubiquitin-based cell cycle indicator to identify compounds that increase cardiac proliferation in embryonic zebrafish. Vitamin D analogs were identified as promoters of embryonic and adult cardiomyocyte proliferation and enhanced injury-induced regeneration, acting locally through cardiac vitamin D receptors. Interestingly, injection of alfacalcidol also increased proliferation in other noncardiac tissues such as retinal cells and hepatocytes, suggesting that vitamin D may be a general regulator of cell division. Loss of vitamin D signaling through generation of vitamin $D$ receptor mutants resulted in decreased

\title{
METALLOPROTEINS
}

\section{A better binder builder}

The design of proteins with novel metal centers generally requires the pre-existence of a suitable binding site in the host scaffold or the use of a consensus sequence derived from natural scaffolds. With the aim of efficiently diversifying the design process, Rittle et al. developed MASCoT (metal active sites by covalent tethering), in which two copies of a stable protein are linked via a disulfide bond to form an interface where a new metal-binding site can be engineered. Using cytochrome $c b_{562}$ as a proof of concept, the authors created a disulfide-linked dimeric protein that binds divalent metal ions in the newly created interface. Further engineering created a small hydrophobic pocket and a high-affinity manganese-binding site. Removal of the heme cofactors from the cytochrome scaffold followed by iron binding in the MASCoT-generated interface demonstrated that the dimeric scaffold can bind ions in multiple oxidation states, and the resulting nonheme iron protein could also bind nitric oxide. MASCOT provides rapid access to potential novel metal-binding sites not found in nature and could help to understand how such sites evolved in biological metalloproteins.

https://doi.org/10.1038/s41589-019-0258-x

length and lack of a proliferative response to alfacalcidol treatment. Finally, RNAsequencing analysis revealed the ErbB2 signaling pathway as a downstream effector of vitamin D, and pharmacological ErbB2 inhibition blocked effects of alfacalcidol. Overall, these findings reveal a new mediator of organ growth and proliferation.

GM

https://doi.org/10.1038/s41589-019-0259-9

\section{TRANSPORTERS}

\section{An appetite for aromatics PLoS Pathog. 15, e1007577 (2019)}

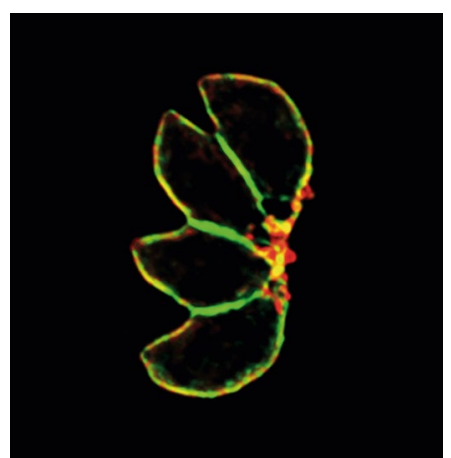

Credit: Giel van Dooren

The Apicomplexa are parasitic protozoa that scavenge essential nutrients from their hosts, generally through transporter proteins. However, very few apicomplexan transporters have been identified and characterized. Parker et al. now identify 66 new polytopic membrane proteins resembling transporters, which they dub ApiATs, in 6 different apicomplexan parasites. Of the 16 ApiATs from Toxoplasma gondii, 10 were expressed in the disease-causing tachyzoite stage of the life cycle, and those that were detectable by immunofluorescence all localized to the parasite periphery. Genetic disruption and mutational analysis implicated three as amino acid transporters, and radioligand quantitation and kinetic assays showed that TgApiAT5-3 exchanges a range of aromatic and large neutral amino acids, with a preference for L-tyrosine. Parasites lacking TgApiAT5-3 demonstrate an inability to transport aromatic amino acids, including L-phenylalanine, which is essential for T. gondii parasite growth in vitro, while work in mice indicates that TgApiAT5-3 is essential for virulence. This work underscores the ability of Apicomplexa to scavenge essential nutrients from their hosts using related transporters.

https://doi.org/10.1038/s41589-019-0260-3

Mirella Bucci, Caitlin Deane, Grant Miura and Yiyun Song 